\title{
Prompt Reconstruction of ATLAS Data in 2010 and 2011
}

\section{Fe}

東京大学 Susumu Oda (ICEPP, University of Tokyo) On behalf of the ATLAS Collaboration

\section{Abstract}

The LHC started operating in November 2009 and has since then provided billions of collision events that were recorded by the ATLAS experiment and promptly reconstructed at an on-site computing farm. The prompt reconstruction chain takes place in two steps and is designed to provide high-quality data for physics publications with as little delay as possible. The first reconstruction step is used for data quality assessment and determining calibration constants and beam spot position, so that this information can be used in the second reconstruction step to optimize the reconstruction performance. After the technical stop of the LHC at the end of 2010, the prompt reconstruction chain had to deal with greatly increased luminosity and pileup conditions. To allow the computing resources to cope with this increased dataflow without developing a backlog, recently significant improvements have been made in the ATLAS reconstruction software to reduce CPU time and file sizes for the produced datasets.

\section{Data taking}

- ATLAS recorded pp collision data at a center of mass energy of $7 \mathrm{TeV}$ with an integrated luminosity of $45 \mathrm{pb}^{-1}$ in 2010 and $>4.8 \mathrm{fb}^{-1}$ in 2011.

- ATLAS data taking is divided into runs (nominally an LHC fill) and Luminosity Blocks ( 2 minutes in 2010 and 1 minute in 2011) over which detector conditions are assumed to be constant.

- The average number of interactions per bunch crossing $\langle\mu\rangle$ was steadily increased to a current maximum of $\sim 15$.

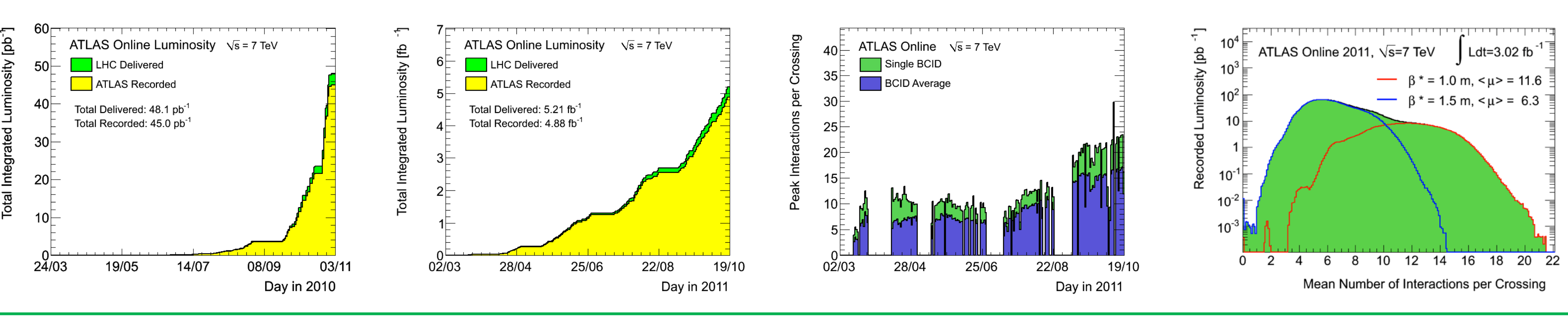

\section{Data format}

- RAW is the output of the ATLAS trigger and data acquisition. File size is reduced using compression by $\sim 50 \%$ and typical size is $\sim 0.7 \mathrm{MB}$ per event in 2011 .

- Event Summary Data contains both detector-level hit information and reconstructed quantities (tracks, calorimeter clusters etc). $\sim 1.5 \mathrm{MB}$ per event

- Analysis Object Data is a subset of ESD and contains the objects and quantities expected to be used by most physics analysis. $200 \mathrm{kB}$ per event

- HIST is ROOT histograms used for data quality monitoring.

- Derived Physics Data is simple ROOT n-tuple used by physics and performance groups.

- TAG contains event-level summary data to enable selection of interesting events for down stream analysis.

Pileup $<\mu>$ dependence of CPU, memory and file size

- Increase of $\langle\mu>$ causes increases of CPU time, memory usage and file size.

- $\langle\mu>$ dependence is monitored, compared with simulation and used to reduce consumptions to efficiently use available computing resources.

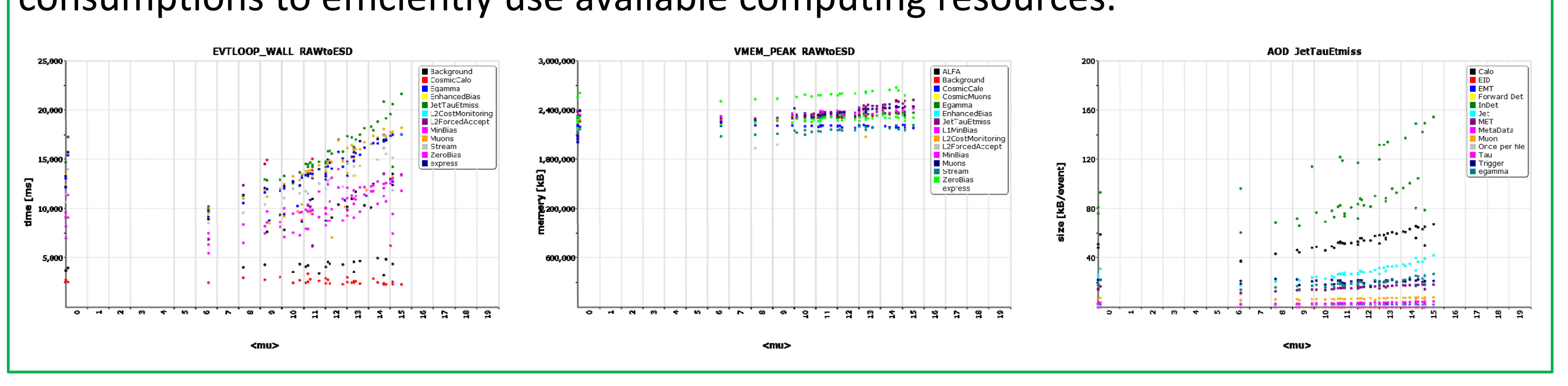

\section{Job monitoring}

- Tier-0 jobs are monitored by shifters and experts using web displays.

- If a problem is found, a report is immediately submitted to the dedicated bug tracker

- The problems is promptly investigated and fixed.

- Fraction of lost events was about $0.02 \%$ for main streams (Egamma, JetTauEtmiss, MinBias and Muons) between March and August in 2011.

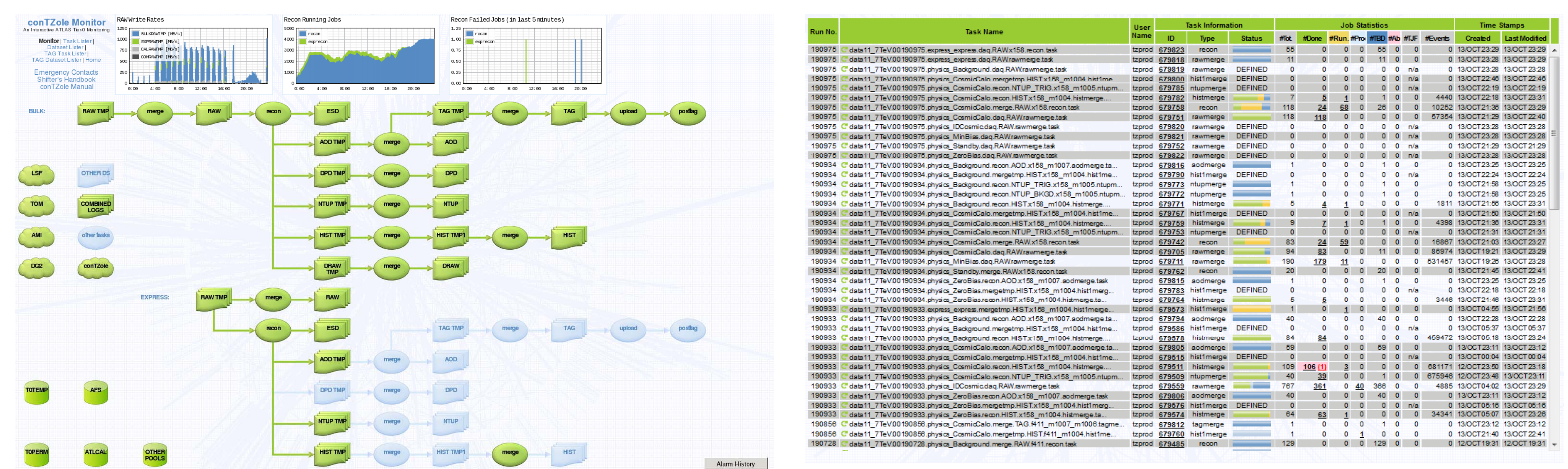

- In 2011 large backlog occurred only at the beginning of August. LHC up-time was $>50 \%$ while the ATLAS computing model assumes up-time of $30 \%$.

The numbers of running and to-be-done jobs in 2011

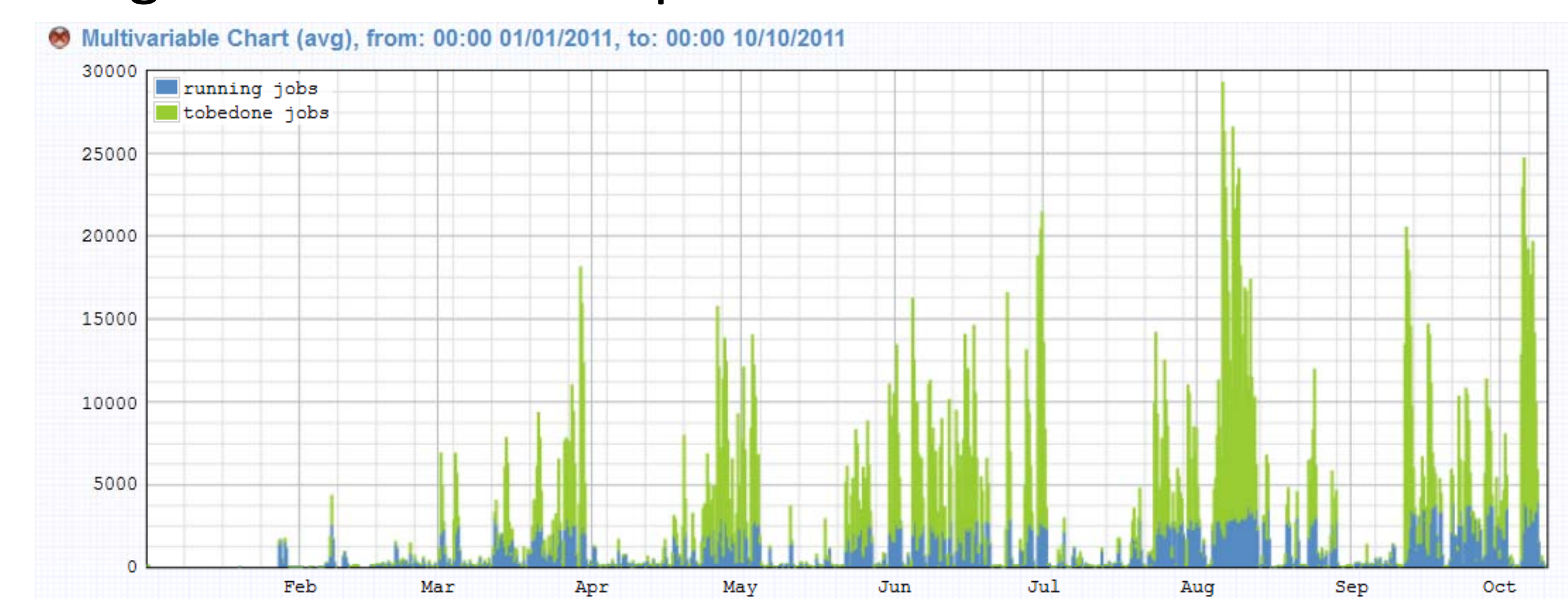

\section{Computing model}

- Computing resources for the ATLAS data reconstruction and analysis are arranged in a hierarchical model with different Tiers.

- Tier-0 is a large computer farm with 3000 dedicated cores at CERN.

- There are 10 Tier-1 centers around the world and many Tier-2 centers organized into clouds around Tier-1s.

- The prompt processing of collision data is performed at Tier-0.

- The outputs are transferred to Tier-1s and finally Tier-2s, over the GRID, for end-user physics analysis.

- Reprocessing of previously recorded data is done at Tier-1s (see N6-4).

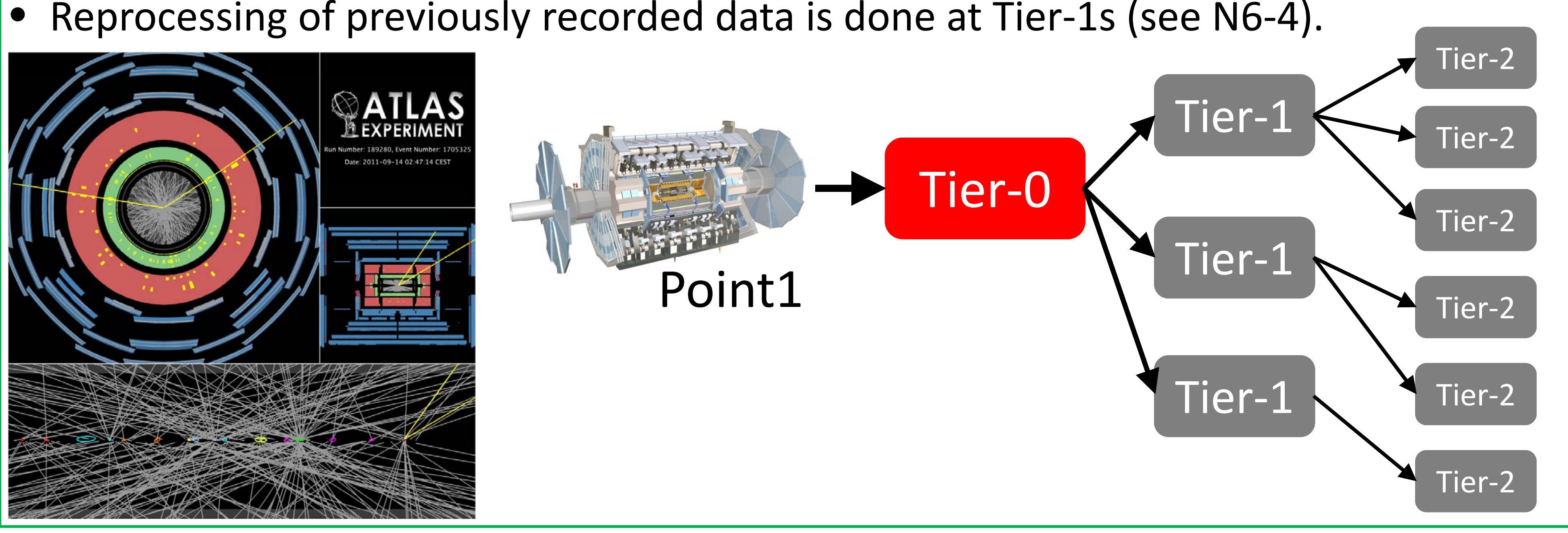

Streaming

- Data are organized into inclusive streams based on trigger chains.

- "Inclusive" means that the same event can appear in more than one stream.

- Express stream is used to check the data quality and calculate calibration constants

in the 48-hour calibration loop. Its rate is about $10 \mathrm{~Hz}$.

- Physics streams consist of Egamma, Muons, JetTauEtmiss and MinBias. The maximum total recording rate is $300-400 \mathrm{~Hz}$.

- Calibration streams contain partial events used by specific ATLAS sub detectors

\section{Time scale}

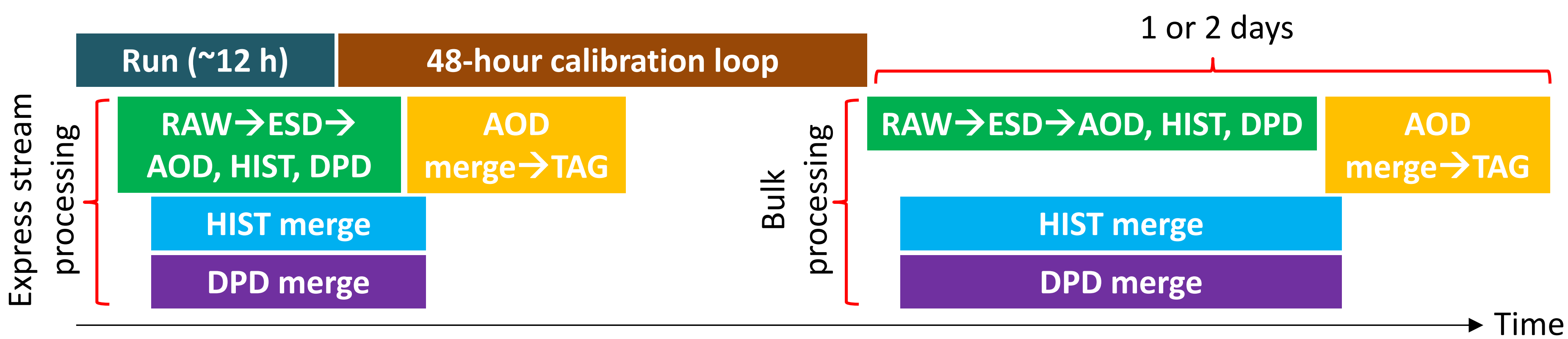

- The time scale at which data are for offline analysis, starting from the moments the collision events occurs at ATLAS.

- Typically, data are available in a form ready for analysis 3 or 4 days after the collisions took place.

\section{Calibration loop}

- Outputs of the express stream processing are sent to the CERN Analysis Facility for analysis (beam spot determination, dead and noisy channel corrections).

- Reconstructed data are assessed by data quality group before the bulk processing starts.

- Calibration constants are uploaded to database, then used in the bulk processing of the physics stream data.

- If calibrations are not ready in time, start of the bulk processing can be delayed by manual intervention. The bulk processing was delayed 10 times in 2011.

\section{Software and testing infrastructure}

- Focus on robustness rather than latest features.

- Production cache is built on top of full ATLAS offline software (Athena) release.

- "Frozen Tier-0" policy allows only changes which do not affect reconstruction in order to be able to combine data of different run periods and to have compatible Monte Carlo samples.

- There are nightly builds of candidate production and development releases, and automatic tests of these builds.

- ATLAS Testing Nightly: run on a very small number of events. There are many of these tests, intended to find crashes when jobs are run with different configurations.

- Run Timer Tester: run on slightly larger samples of events, and more tools are

available to analyze the output of the job.

- Tier-0 Chain Test: run the full Tier-0 workflow, from RAW input, to produce ESD, AOD and TAG files, and merge them. This test is also used to enforce the Frozen Tier-0 policy by performing a byte-by-byte comparison of the outputs of two successive nights.

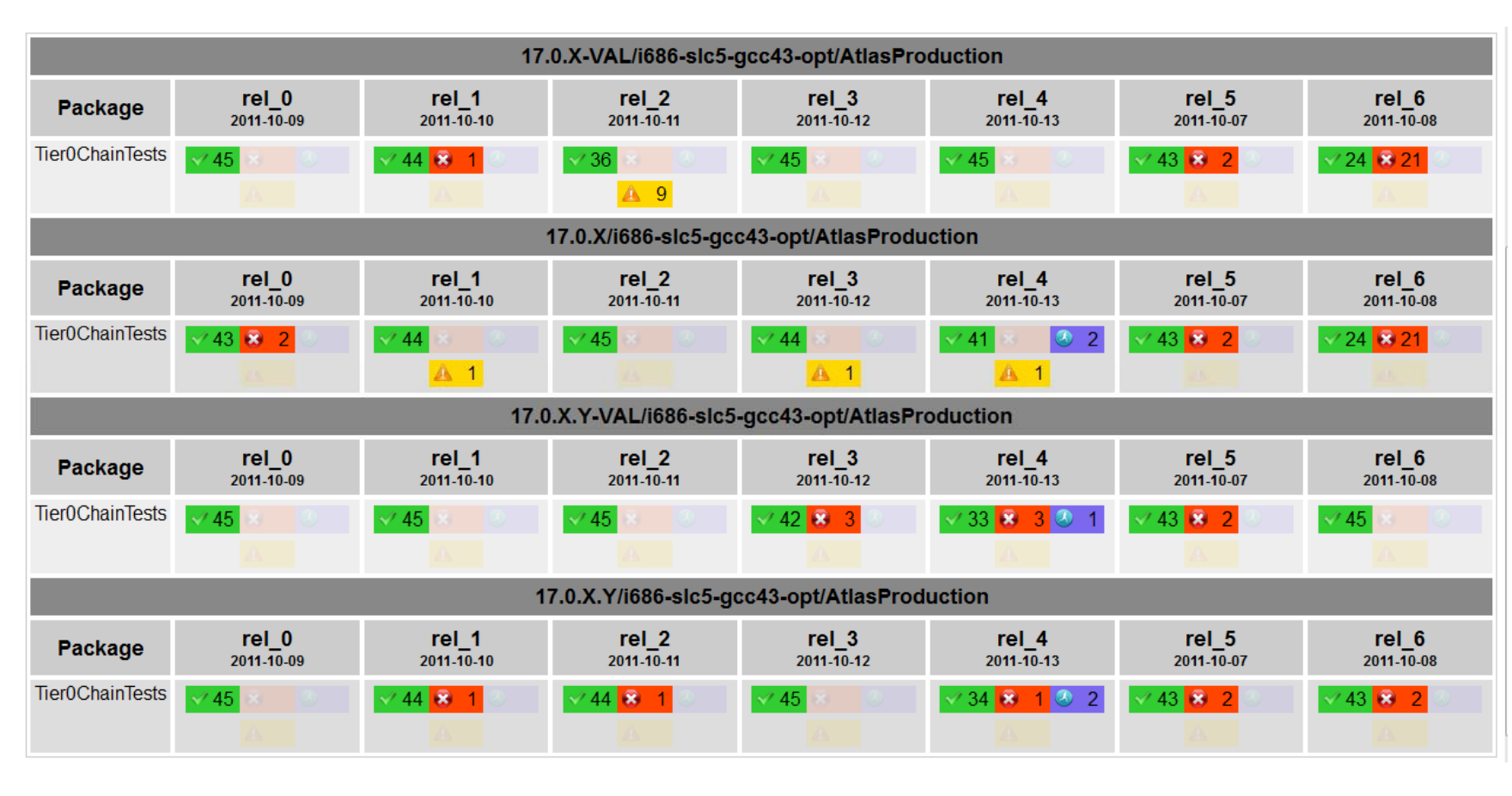

\section{Physics results}

- ATLAS public results are available at https://twiki.cern.ch/twiki/bin/view/AtlasPublic - Results shown at summer conferences in 2011 were based on the prompt processing at Tier-0. - Analysis for 2012 winter conferences will use both datasets by the reprocessing at Tier- 1 s and the prompt processing at Tier- 0 .

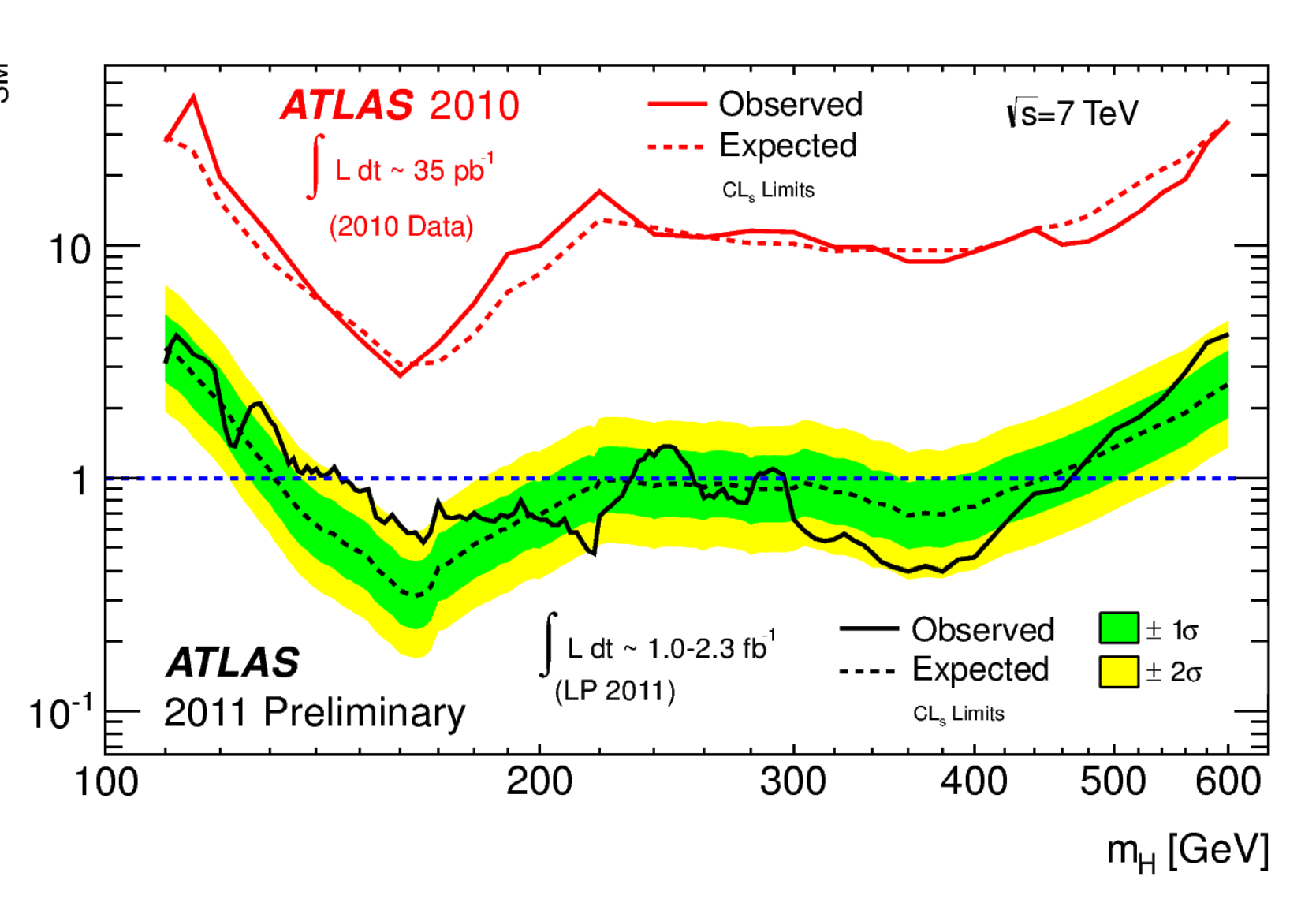

